Noname manuscript No.

(will be inserted by the editor)

\title{
A multi-electrode array and inversion technique for retrieving six conductivities from heart potential measurements
}

\author{
Barbara M. Johnston - Peter R. Johnston
}

Received: date / Accepted: date

\begin{abstract}
A method for accurately finding cardiac bidomain conductivity parameters is a crucial part of efforts to study and understand the electrical functioning of the heart. The bidomain model considers current flowing along (longitudinal) and across (transverse) sheets of cardiac fibres, as well as between these sheets (normal), in both the extracellular and intracellular domains, which leads to six conductivity values. To match experimental studies such a method must be able to determine these six conductivity values, not just the four where it is assumed that the transverse and normal conductivities are equal. This study presents a mathematical model, solution technique, multi-electrode array and two-pass inversion method, which can be used to retrieve all six conductivities from measurements of electrical potential made on the array. Simulated measurements of potential, to which noise is added, are used to demonstrate the ability of the method to retrieve the conductivity values. It is found that not only is it possible to accurately retrieve all six conductivity values, as well as a value for fibre rotation angle, but that the accuracy of such retrievals is comparable to the accuracy found in a previous study when only four conductivities (and fibre rotation) were retrieved.
\end{abstract}

Keywords bidomain model · cardiac conductivity values · anisotropy · simulation · electrode array

\section{Introduction}

It is well-recognised [2] that accurate measurements of cardiac conductivity values are fundamental for use in models that study the electrical conductivity of the

B. Johnston

Queensland Micro- and Nanotechnology Centre, School of Biomolecular and Physical Science, Griffith University, Nathan, Queensland, Australia, 4111

Tel.: +61-7-37354405

Fax: +61-7-37357656

E-mail: : Barbara.Johnston@griffith.edu.au.

P. Johnston

Queensland Micro- and Nanotechnology Centre, School of Biomolecular and Physical Science, Griffith University, Nathan, Queensland, Australia, 4111 
heart, since, in certain areas, models are able to facilitate understanding that cannot be gained from simply making experimental measurements. These areas include, for example, the mechanics of defibrillation [2] or an understanding of ST segment deviation [26]. In particular, electrical anisotropy is critically important in both initiating and maintaining re-entrant arrhythmia and in determining the effectiveness of cardioversion [1].

Many of these studies use the bidomain model [31] to model the electrical behaviour of the cardiac tissue. This regards the cardiac tissue as consisting of two interpenetrating domains, extracellular $(e)$ and intracellular $(i)$, in which the cardiac properties are averaged. In addition, cardiac tissue is laminar in nature and consists of sheets of cardiac fibres that rotate in orientation between the inner and outer heart surface. These models assume that current can flow in three directions, along the fibres (longitudinal $l$ ), normal to this within the sheet (transverse $t$ ) and normal $(n)$ to the sheet of fibres, giving six different cardiac conductivity values in the model, $g_{i l}, g_{i t}, g_{i n}, g_{e l}, g_{e t}$ and $g_{e n}$. However, in order to make the problem more tractable, such studies often assume that the cardiac conductivities normal to the fibres in the plane of the fibre sheet are equal to those which are in the direction that is normal to the sheet, leaving four conductivity values $g_{i l}, g_{i t}, g_{e l}$ and $g_{\text {et }}$.

Few measurements of cardiac conductivity values have ever been made [3,29, 28], due both to the experimental difficulty of making such measurements and also the interpretation of the measurements. The few sets of four conductivity values that do exist vary considerably in value and in their effect when used in modelling studies $[30,18,17]$. Recent experimental work $[9,11,1]$ has questioned the validity of this assumption and this has been supported by recent simulation work [16], which found that there are significant differences between heart surface potential distributions produced using four conductivity values and those produced using six values, and that the six conductivity distributions are a better match for experimental results. Neither of the two available six conductivity datasets $[22,12]$ that is used in this study has been fully experimentally determined.

The inverse problem of inferring conductivities from measurements of electric potential is highly ill-posed and computationally intensive [7]. Recent work in this area includes a proposed method that maps the electrical activation of cardiac tissue and then uses a least squares and singular value decomposition approach to obtain the optimum set of cardiac parameters [8]. A new suggested approach [7], applied to a $2 \mathrm{D}$ monodomain, is to simplify the problem into a set of computationally tractable problems via a single-step approximation. Other recent work in this area includes an investigation into the sensitivity of the forward problem of electrocardiology to the tissue conductivity parameters associated with the various organs in the human thorax [6].

Another aspect of recent work is research into the design and fabrication of micro-electrodes for use in studies associated with the measurement of cardiac conductivity values. Hooks and Trew [10] designed, fabricated and validated a plunge electrode array, which contained 137 recording sites for potential via needles that housed 13 silver wires within a total diameter of $0.7 \mathrm{~mm}$. This array was then used to test an approximate relationship between the four bidomain conductivities and the monodomain conductivities measured by the array. An extension of this work [1] used a 325 electrode array with an approximate spacing of $4 \times 4 \times 1 \mathrm{~mm}$ and measurements using porcine left ventricular tissue to demonstrate 
that ventricular tissue is electrically orthotropic with three distinct propagation directions. A recent different approach [25,24] proposes using multisite interstitial stimulation and recording via a network of very small $12.5 \times 12.5 \times 12.5 \mu \mathrm{m}^{3}$ MEMS fabricated blocks, in conjunction with Bayesian mathematical methods to obtain microimpedances, under the assumption that normal and transverse values are equal.

Previous work in this area by the authors and others $[15,13,14]$ involved the design of a two-layer multi-electrode array, which was used to show that four cardiac conductivity values, as well as a value for fibre rotation angle could be retrieved from simulated measurements of potential on the heart surface. That work introduced a mathematical model, a solution method and an inversion procedure, as well as the electrode array, and investigated the accuracy of the retrievals. The present work aims to extend the mathematical model and its solution method, as well as the inversion procedure, to allow for the retrieval of six rather than four conductivity values (in addition to fibre rotation). To our knowledge, such a technique has not been demonstrated previously. The method will include the design of a new multi-electrode array, suitable for making the measurements.

\section{Model and Solution Method}

\subsection{Governing Equations}

The bidomain governing equations [31] for the potentials in cardiac tissue are

$$
\nabla \cdot \mathbf{M}_{i} \nabla \phi_{i}=\frac{\beta}{R}\left(\phi_{i}-\phi_{e}\right) \quad \text { and } \quad \nabla \cdot \mathbf{M}_{e} \nabla \phi_{e}=-\frac{\beta}{R}\left(\phi_{i}-\phi_{e}\right)-I_{s}
$$

where $i=$ intracellular, $e=$ extracellular, the tensors $\mathbf{M}_{j}(j=i$ or $e)$ are discussed below and $\phi_{j}(j=i$ or $e)$ is the potential, $\beta$ is the surface to volume ratio of the cells, $R$ is the specific membrane resistance and $I_{s}$ is the external current source per unit volume applied in the extracellular space.

The tensors $\mathbf{M}_{j}(j=i$ or $e$ ) take account of the anisotropic nature of the tissue, which is related to the varying conductivity values, as well as to fibre rotation within the cardiac tissue [18]. Since the current flows at a different speed in three directions, along the fibres within the sheet (longitudinal $=l$ ), normal to the fibres within the sheet (transverse $=t$ ) and normal to the sheets of fibres (normal $=n$ ) and this takes place in the two domains (extracellular $=e$ ) and (intracellular $=i)$, the bidomain model requires six conductivity values, $g_{i l}, g_{i t}, g_{i n}, g_{e l}, g_{e t}$ and $g_{e n}$. Assuming that $g_{i n}=g_{i t}$ and $g_{e n}=g_{e t}$ leads to a four-conductivity dataset. However, in this work this assumption will not be made and the model will be solved using all six bidomain conductivities.

The potential distribution in the blood, $\phi_{b}$, is governed by Laplace's equation

$$
\nabla^{2} \phi_{b}=0 \text {. }
$$

\subsection{Geometry of the Model}

This model considered in this work is a block of ventricular cardiac tissue that has dimensions $2 \mathrm{~cm} \times 2 \mathrm{~cm}$ in the $x-y$ plane and which represents the outer surface of 


\begin{tabular}{|c|c|}
\hline Tissue & $z=0$ \\
\hline Blood & $z=1$ \\
\hline
\end{tabular}

Fig. 1 Cross-sectional view of the tissue-blood model used here. The tissue lies between the epicardium $(z=0)$ and the endocardium $(z=1)$, with the blood in the region $z>1$.

the heart (epicardium). It also extends $1 \mathrm{~cm}$ in the $z$ direction to the inner surface of the heart (endocardium) at $z=1$, which is in contact with a volume of blood, which extends to infinity in the positive $z$ direction (Figure 1).

As in previous studies $[13,15]$, it will be assumed that the rotation of the main fibre axis between the epicardium and the endocardium varies linearly with depth through an angle $\alpha$. Hence $\mathbf{M}_{j}$, for a rectilinear block of tissue, is of the form

$$
\mathbf{M}_{j}(x, y, z)=\left(\begin{array}{ccc}
\left(g_{j l}-g_{j t}\right) c^{2}+g_{j t} & \left(g_{j l}-g_{j t}\right) c s & 0 \\
\left(g_{j l}-g_{j t}\right) c s & \left(g_{j l}-g_{j t}\right) s^{2}+g_{j t} & 0 \\
0 & 0 & g_{j n}
\end{array}\right)
$$

where $j=i$ or $e, c=\cos \alpha z$ and $s=\sin \alpha z$.

\subsection{Boundary Conditions}

Assuming the epicardium is insulated and, since there is continuity of potential and current at the interface between the tissue and the blood, but the intracellular space is insulated by the extracellular space, the boundary conditions used to solve the model include

$$
\frac{\partial \phi_{e}}{\partial z}=\frac{\partial \phi_{i}}{\partial z}=0 \quad \text { at } z=0
$$

and

$$
\phi_{e}=\phi_{b}, \quad g_{b} \frac{\partial \phi_{b}}{\partial z}=g_{e n} \frac{\partial \phi_{e}}{\partial z}, \quad \frac{\partial \phi_{i}}{\partial z}=0 \quad \text { at } z=1
$$

where $g_{b}$ is the conductivity of blood and $\phi_{b}$ is the potential in the blood. Also, $\phi_{b} \rightarrow 0$ as $z \rightarrow \infty$, since the blood mass is assumed infinite in the positive $z$ direction. Finally, assuming that the boundaries of the domain are insulated, gives at the $x$ and $y$ boundaries

$$
\mathbf{M}_{e} \nabla \phi_{e} \cdot \mathbf{n}=0, \mathbf{M}_{i} \nabla \phi_{i} \cdot \mathbf{n}=0 \text { and } \nabla \phi_{b} \cdot \mathbf{n}=0 .
$$

where $\mathbf{n}$ is the outward pointing normal from the boundary.

\subsection{Solution Technique}

The solution technique for the forward model, equations (1)-(2), subject to boundary conditions (4)-(6), first involves expansion of $\phi_{i}$ and $\phi_{e}$ as a Fourier series (see 
Table 1 Conductivity data (in $\mathrm{mS} \mathrm{cm}^{-1}$ ) and space constants (in $\mathrm{mm}$ ) from the indicated studies.

\begin{tabular}{c|cccccc|ccc}
\hline Study & $g_{e l}$ & $g_{\text {et }}$ & $g_{\text {en }}$ & $g_{i l}$ & $g_{i t}$ & $g_{\text {in }}$ & $\lambda_{l}$ & $\lambda_{t}$ & $\lambda_{n}$ \\
\hline MacLachlan et al. [22] & 2.0 & 1.65 & 1.35 & 3.0 & 1.0 & 0.35 & 0.74 & 0.53 & 0.36 \\
Hooks et al. [9] & 2.63 & 2.45 & 1.087 & 2.63 & 0.263 & 0.08 & 0.77 & 0.33 & 0.18 \\
\hline
\end{tabular}

[15] for more detail)

$$
\begin{array}{r}
\phi_{j}(x, y, z)=\sum_{n=0}^{\infty} \sum_{m=0}^{\infty} C_{n m}^{j}(z) \cos (m \pi y) \cos (n \pi x)+D_{n m}^{j}(z) \sin (m \pi y) \cos (n \pi x) \\
\quad+E_{n m}^{j}(z) \cos (m \pi y) \sin (n \pi x)+F_{n m}^{j}(z) \sin (m \pi y) \sin (n \pi x)
\end{array}
$$

where $j=i$ or $e$. These expansions are substituted into equations (1) to give two sets of four ordinary differential equations in the coefficients $C_{n m}^{j}(z), D_{n m}^{j}(z)$, $E_{n m}^{j}(z)$, and $F_{n m}^{j}(z)$. These coefficients are found via a one-dimensional finite difference scheme that leads to a banded system of linear algebraic equations, which can be solved numerically for $C_{n m}^{j}(z), D_{n m}^{j}(z), E_{n m}^{j}(z)$, and $F_{n m}^{j}(z)$ using standard techniques [27]. The series in equation (7) is then summed to give the potentials. Thus the potential at any point in the domain (for example, at an electrode) can be obtained by summing the series there.

\subsection{Parameters Used in the Model}

Two sets of conductivity values are used to solve the model, those of MacLachlan et al. [22], which are based on the work of Foster and Schwan [4], and those of Hooks et al. [12] (Table 1). Other parameters are taken to be [14] $\beta=2000$ $\mathrm{cm}^{-1}, R=9100 \Omega \mathrm{cm}^{2}, I_{S}=50 \mu \mathrm{A} \mathrm{mm} \mathrm{m}^{-3}$ and $g_{b}=6.7 \mathrm{mS} \mathrm{cm}^{-1}$. This gives space constants $\left.\left(\lambda_{q}=\sqrt{\frac{R}{\beta}\left(\frac{g_{i q} g_{e q}}{g_{i q}+g_{e q}}\right.}\right), q=l, t, n\right)$ in the various directions in the range 0.18 $\mathrm{mm}-0.77 \mathrm{~mm}$ (Table 1 ). The fibre rotation angle $\alpha$, which describes the rotation of the fibre axis between the epicardium and the endocardium, is taken to be $\frac{2 \pi}{3}$, to be consistent with previous studies $[13,14]$.

\section{Methods}

\subsection{Inversion Process}

In the forward model, described by equations (1)-(2), the potentials depend on the required parameters in a nonlinear fashion,

$$
\mathbf{G}(\mathbf{m})=\mathbf{\Phi}
$$

where $\boldsymbol{\Phi}$ is the vector of electrode potentials, $\mathbf{m}=\left[g_{i l}, g_{i t}, g_{i n}, g_{e l}, g_{e t}, g_{e n}, \alpha\right]^{T}$ and $\mathbf{G}$ represents the model. Under experimental conditions there will be noise in the measurement vector $\boldsymbol{\Phi}$, so to obtain an approximation to $\mathbf{m}$ from equation (8) it is necessary to minimise the Tikhonov functional

$$
\|\mathbf{G}(\mathbf{m})-\mathbf{\Phi}\|_{2}^{2}+\gamma^{2}\|\mathbf{m}\|_{2}^{2}
$$


Here $\gamma$ is the regularisation parameter.

Minimisation of the functionals is performed using the SolvOpt solver [19], which minimises non-linear multivariate functions using a modified Shor's $r$ algorithm. The constraints applied for the minimisation are that $0 \leq \alpha \leq \pi$ and that the conductivities are positive. The termination criteria used [19] is that the relative error in the functional is less than $10^{-6}$ for two successive iterations.

Percentage relative error is used to compare the parameters $p_{c}$, found during the inversion, with the generating parameters $p$ from Section 2.4, where

$$
\text { Percentage relative error }=\left|\frac{p-p_{c}}{p}\right| \times 100 \%
$$

\subsection{Proposed Electrode Array and Retrieval Method}

The electrode array proposed in this work is an extension of an array already published by the authors and others [14], which was successful in retrieving the four bidomain conductivities $\mathrm{g}_{i l}, \mathrm{~g}_{i t}, \mathrm{~g}_{e l}$ and $\mathrm{g}_{e t}$ from simulated measurements of potential, as well as fibre rotation angle $\alpha$. It consists of 17 micro-needles (Figure 2), each containing three electrodes, situated in three parallel 'layers' (Figure 3). Two electrodes are used for current injection and the potentials, measured on subsets (Figure 3) of the remaining electrodes, are used to find the cardiac bidomain parameters.

The dimensions of the array $(0.5 \mathrm{~mm}$ between needles and also between electrodes on each needle) are chosen to be consistent with making potential measurements at distances less than or equal to the space constant (Table 1) of the tissue ('closely spaced'), as well as at distances greater than the space constant ('widely spaced'). This is in accord with the four-electrode technique of Plonsey and Barr [23], who demonstrated theoretically that four collinear equally spaced electrodes, with source and sink on the outer electrodes and inner measuring electrodes, placed both along and across the cardiac fibres, required both 'closely' and 'widely spaced' measurements to isolate the extracellular and intracellular conductivities. The placement of the source and sink electrodes here is also chosen in accord with that work, and, for the 'closely-spaced' electrode set, it is the same as in the array used in the previous work [14]. However, because of the extra layer of electrodes in the 'widely-spaced' set, a number of choices could be made for the positions of the source and sink and the positions that are proposed here are the ones that appear to work well, rather than ones that are guaranteed to be placed in the optimal positions.

It is found, similarly to the previous work, that a two-pass protocol is needed to find the desired parameters accurately [14] using the one array. The extracellular conductivities are found on the first pass, using a subset (Figure 3(a)) of electrodes that are 'closely spaced' $(0.5 \mathrm{~mm})$ and then a subset (Figure $3(\mathrm{~b}))$ of 'widely' spaced electrodes (a mixture of $0.5 \mathrm{~mm}$ and $1 \mathrm{~mm}$ spacing) are used in the second pass. The extracellular conductivities from the first pass are held constant in the second pass, which retrieves the intracellular conductivities [23] (and fibre rotation angle). 


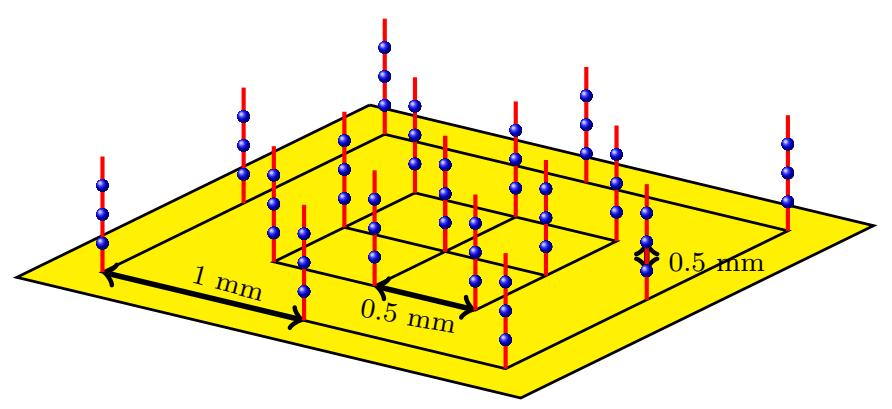

Fig. 2 Proposed three-layer multi-electrode array used to retrieve six conductivity values. Subsets of the electrodes (see Figure 3) are used in the inversion method.

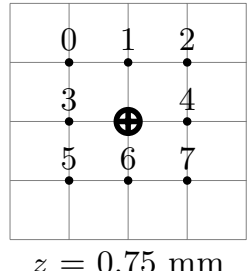

$z=0.75 \mathrm{~mm}$

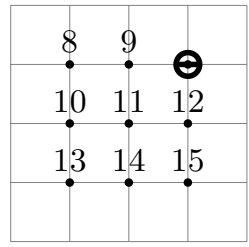

$z=1.25 \mathrm{~mm}$

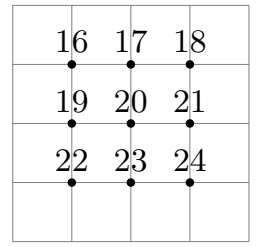

$z=1.75 \mathrm{~mm}$

(a) 'Closely-spaced electrode set
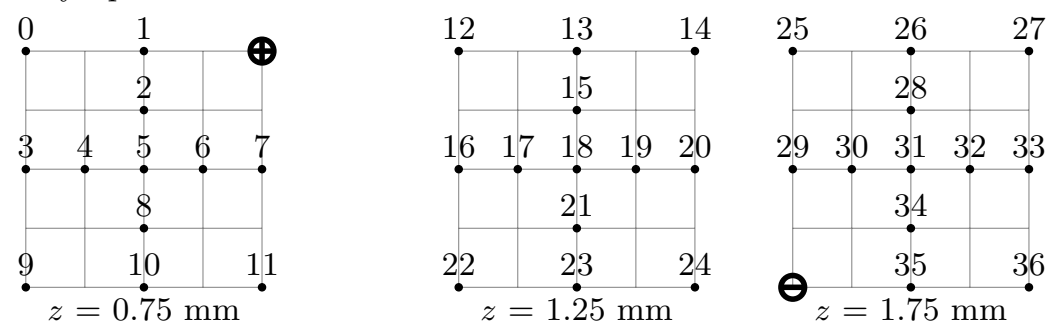

(b) 'Widely-spaced electrode set

Fig. 3 Plan view of the three layers of the multi-electrode array, shown in Figure 2, that are used in (a) the first and (b) the second pass of the protocol to retrieve the six conductivities. The spacing between electrodes is $0.5 \mathrm{~mm}$ in all directions in the first pass, as well as in the normal direction in both passes. The spacing between each of the marked squares is also 0.5 $\mathrm{mm}$, resulting in a mixture of $1 \mathrm{~mm}$ and $0.5 \mathrm{~mm}$ spacing in both the $l$ and $t$ directions in the second pass. Source and sink electrodes for addition and removal of the applied current are indicated by + and - respectively.

\subsection{Comparison of the proposed array and the previous array}

The electrode array, proposed in Section 3.2, is a three layer analogue of the array used previously $[13,14]$ to retrieve the four conductivities, $\mathrm{g}_{i t}, \mathrm{~g}_{i t}, \mathrm{~g}_{e l}$ and $\mathrm{g}_{e t}$. The new array has simply had a third layer of electrodes added, with the distance between electrodes in the normal direction now $0.5 \mathrm{~mm}$ instead of $0.3 \mathrm{~mm}$. Both arrays have 17 micro needles, with each needle in the proposed array containing 3 electrodes, rather than 2 electrodes as previously. 
When simulations were conducted with the old two-layer array to attempt to find the six bidomain conductivities (that is, $\mathrm{g}_{i n}$ and $\mathrm{g}_{e n}$ in addition to the four mentioned above), it was found that it was not possible to retrieve $g_{i n}$ and that an additional layer of elecrodes was necessary. It is not surprising that more than two electrodes are required in the normal direction, given that this method is based on the four-electrode technique. However, it is interesting that three rather than four electrodes per needle are sufficient.

While the proposed 'closely-spaced' electrode set (Figure 3(a)) is identical to the previous set [14], except for the fact that it now has a third 'layer' of electrodes, this is not the case for the proposed 'widely-spaced' electrode set (Figure 3(b)). This is because testing with the previous set that simply had a third layer of electrodes added found that that subset was unable to retrieve the intracellular conductivities with sufficient accuracy and that it was necessary to add some extra electrodes, for example, electrodes \# 2, 4, 6 and 8 in the $z=0.75 \mathrm{~mm}$ layer and their counterparts in the second and third layers.

\subsection{First Pass}

The bidomain conductivity values of MacLachlan et al. [22], together with the parameters given in Section 2.5, are used to produce a set of simulated potential measurements on the 'closely-spaced' subset of electrodes in the proposed array (Figure $3(\mathrm{a})$ ). Noise of $1 \%, 2 \%$ and $5 \%$ is added to these measurements and the inversion process described in Section 3.1 is then used to retrieve $g_{e l}, g_{e t}, g_{e n}, g_{i l}, g_{i t}, g_{i n}$ and $\alpha$ simultaneously during the first pass through the solver.

In this case the Tikhonov functional, from equation (9), to be minimised is

$$
f_{1}=\sum_{i=0}^{24}\left[\phi_{M}(i)-\phi_{C}(i)\right]^{2}+\gamma_{1}^{2}\left[g_{i l}^{2}+g_{i t}^{2}+g_{i n}^{2}+g_{e l}^{2}+g_{e t}^{2}+g_{e n}^{2}\right]+\gamma_{2}^{2} \alpha^{2}
$$

where $\phi_{M}$ is the difference in measured potential between electrode $i$ and the reference electrode and $\phi_{C}$ is similar but for the calculated value at each iteration of the solver. Here $\mathbf{m}=\left[g_{i l}, g_{i t}, g_{i n}, g_{e l}, g_{e t}, g_{e n}, \alpha\right]^{T}$, and, as in the previous work [15], the two regularisation parameters, $\gamma_{1}$ and $\gamma_{2}$ are taken to be $10^{-2}$ and $10^{-5}$, respectively. These were chosen in the previous work [15] because of the difference in the magnitudes of the conductivity values and $\alpha$. Testing in the current work found that results from both passes of the new method were quite insensitive to changes in the regularisation parameters and so it was decided to retain the values used previously. The sensitivity of the results to the starting value in the first pass was also investigated. It was found that using initial values that were all either 10 times smaller or 10 times larger than the usual initial values made no difference to the values retrieved by the solver. Initial values used in the first pass are $\alpha=0.5$, with the conductivity values all equal to $1 \times 10^{-3}$, except for $g_{i n}=1 \times 10^{-4}$. A set of 10 first passes is produced for each noise level, to simulate 10 different experiments. 


\subsection{Second Pass}

A similar set of simulated potentials is produced on the 'widely-spaced' electrode subset (Figure 3(b)), to which noise is added before the inversion takes place. However, for the second pass, the mean values for $g_{e l}, g_{e t}$ and $g_{e n}$ from the first pass are held constant and this time only values for $g_{i l}, g_{i t}, g_{i n}$ and $\alpha$ are retrieved, using starting values which are their means from the first pass. This means that the Tikhonov functional to be minimised is now

$$
f_{2}=\sum_{i=0}^{36}\left[\phi_{M}(i)-\phi_{C}(i)\right]^{2}+\gamma_{1}^{2}\left[g_{i l}^{2}+g_{i t}^{2}+g_{i n}^{2}\right]+\gamma_{2}^{2} \alpha^{2}
$$

Again a set of 10 passes is made for each noise level. Finally, the entire process is repeated for the data of Hooks et al. [9] (Table 1).

\subsection{Exclusion of 'Non-physiological' Second Pass Runs}

In the first pass of the retrieval protocol, it was sometimes the case that one (and occasionally two) of the intracellular conductivities converged to values that were non-physiological (for example, $\mathrm{O}\left(10^{-9}\right)$ ). This was dealt with by running more than the required 10 inversions and calculating the mean and standard deviation over all these inversions for each of the seven parameters to be retrieved (three extracellular and three intracellular conductivities and fibre rotation angle). Particular inversion runs were then excluded if they had three more parameters which lay outside of the mean \pm 1 standard deviation range.

\section{Results}

Plots for percentage relative error, versus the iteration number in the SolvOpt solver [19], for a typical example where $1 \%$ noise is added, are given in Figure 4. In part (a) of the figure, the extracellular conductivities are retrieved in 92 iterations, while the second pass to retrieve the intracellular conductivities and the fibre rotation angle, shown in the second part of (b) and (c), respectively, takes an additional 38 iterations. Note that the jump in conductivities at iteration 93 of Figure 4(b) is related to the start of the second pass and is not significant. The extracellular conductivities are retrieved very accurately in the first pass, dropping below the added noise after about 70 iterations. At that stage, the relative errors for the intracellular conductivities are still above $1 \%$, but these drop down during the second pass as do the values for the error in the fibre rotation angle (the right-hand sides of the plots in (b) and (c), respectively).

Typically around 80 iterations are required in the first pass, where the six conductivity values $\left(\mathrm{g}_{j k}, j=i, e, k=l, t, n\right)$ and the fibre rotation are retrieved (Section 2.4) and then approximately a further 40 iterations are needed to accurately retrieve the intracellular and fibre rotation angle values. This compares with around 60 followed by 30 iterations for the previous two layer array, where only four conductivity values as well as fibre rotation were retrieved.

Percentage relative errors \pm one standard deviation, averaged over 10 runs (Section 3.2), for the noise levels 1\%, 2\% and 5\%, are given in Table 2, for the 


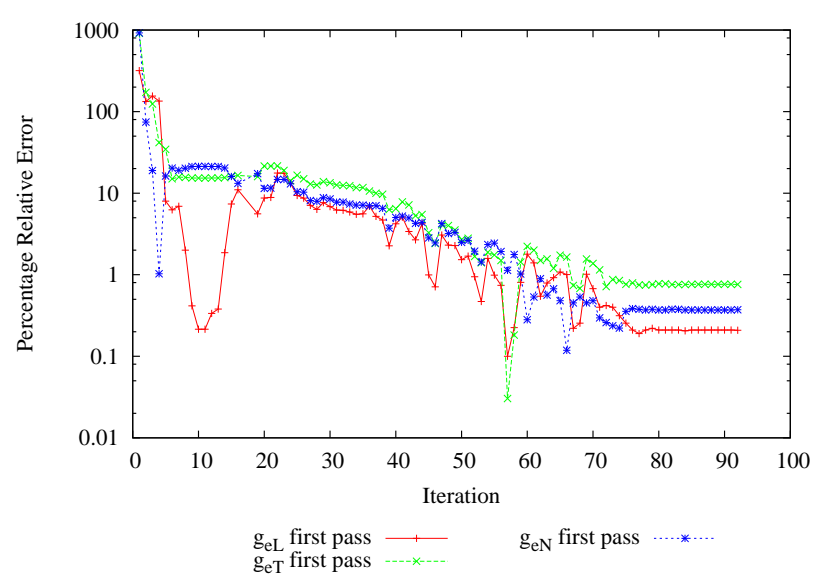

(a) Extracellular conductivities

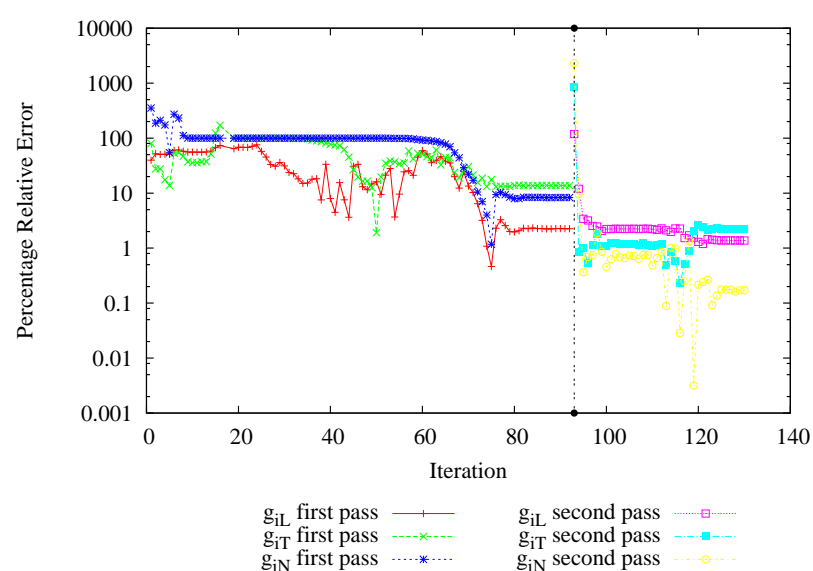

(b) Intracellular conductivities

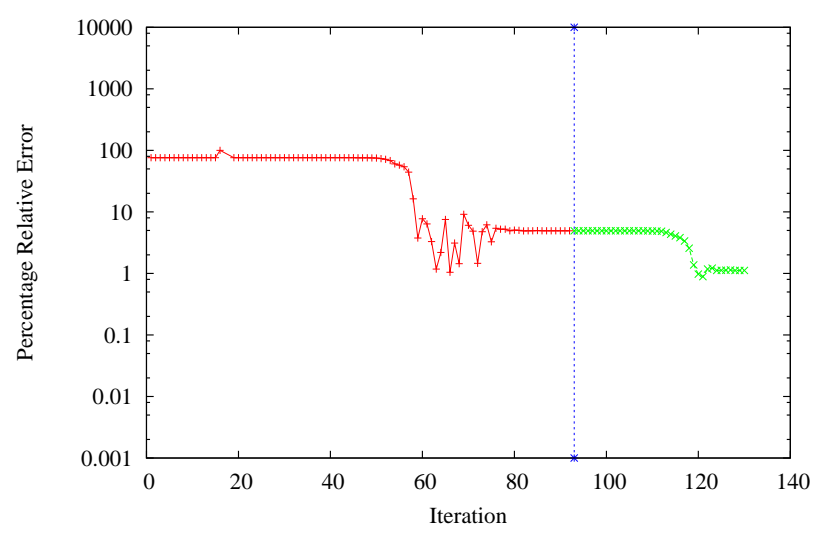

first pass -1 second pass $-\cdots-\cdots$

(c) Fibre rotation angle

Fig. 4 An example, for $1 \%$ noise added, of percentage relative errors for the (a) extracellular conductivities, (b) intracellular conductivities and (c) fibre rotation angle, versus the iteration number in the SolvOpt [19] algorithm. Extracellular conductivities are retrieved in the first pass and intracellular conductivity and fibre rotation angle values from the first pass are refined in the second pass. The vertical line in (b) and (c) separates the first from the second pass. Note the differing vertical scales. 
Table 2 Average percentage relative errors \pm 1 standard deviation, for various noise levels, when recovering the cardiac parameters for the indicated datasets. The extracellular conductivities are retrieved in the first pass and the intracellular conductivities and fibre rotation angle are retrieved in the second pass.

\begin{tabular}{c|ccc|ccc}
\hline & \multicolumn{2}{|c}{ MacLachlan et al. [22] } & \multicolumn{3}{c}{ Hooks et al. [9] } \\
Noise & $1 \%$ & $2 \%$ & $5 \%$ & $1 \%$ & $2 \%$ & $5 \%$ \\
\hline$g_{e l}$ & $0.2 \pm 0.2$ & $0.5 \pm 0.8$ & $0.3 \pm 1.1$ & $0.1 \pm 0.3$ & $0.2 \pm 0.5$ & $1.1 \pm 1.8$ \\
$g_{\text {et }}$ & $0.4 \pm 0.5$ & $0.1 \pm 1.3$ & $0.3 \pm 1.9$ & $0.1 \pm 0.4$ & $0.2 \pm 0.9$ & $0.5 \pm 2.4$ \\
$g_{e n}$ & $0.1 \pm 0.2$ & $0.1 \pm 0.6$ & $0.3 \pm 1.3$ & $0.1 \pm 0.3$ & $0.2 \pm 0.6$ & $0.4 \pm 0.8$ \\
\hline$g_{i l}$ & $1.2 \pm 1.1$ & $3.5 \pm 2.0$ & $1.1 \pm 5.5$ & $0.2 \pm 0.7$ & $0.7 \pm 2.3$ & $6.5 \pm 6.1$ \\
$g_{i t}$ & $3.8 \pm 2.3$ & $0.3 \pm 3.1$ & $2.9 \pm 9.1$ & $1.4 \pm 2.7$ & $10.2 \pm 5.9$ & $7.4 \pm 13.6$ \\
$g_{i n}$ & $0.6 \pm 1.3$ & $0.9 \pm 3.8$ & $6.9 \pm 7.5$ & $3.8 \pm 3.8$ & $3.4 \pm 4.3$ & $15.6 \pm 17.5$ \\
\hline$\alpha$ & $1.7 \pm 1.3$ & $1.2 \pm 2.7$ & $1.8 \pm 6.5$ & $0.4 \pm 0.6$ & $2.0 \pm 1.6$ & $0.3 \pm 5.6$
\end{tabular}

datasets of MacLachlan et al. [22] and Hooks et al. [9] (Table 1). The extracellular values are the mean of the 10 first pass inversions and the intracellular and fibre rotation values are the means of the second pass inversions. The errors in Table 2 show that, not only is it possible to retrieve all six conductivity values $\left(\mathrm{g}_{j k}\right.$, $j=i, e, k=l, t, n)$, as well as fibre rotation, it is also possible to do so very accurately. This is so particularly in the case of the extracellular conductivities, where the average percentage relative errors are generally less than one quarter of the added noise, with similar values for both datasets. It is worth noting that the accuracy, for the extracellular conductivities, does not appear to fall off, relative to the noise, as the noise increases.

The intracellular values are not retrieved as accurately as the extracellular values, but the errors still compare favourably with the errors found previously [14] with the two layer array, where only $g_{i t}$ and $g_{e t}$ (and $\alpha$ ) were being retrieved. For example, for $1 \%$ noise and the previous two layer array [14], the percentage relative errors for $g_{i l}$ and $g_{i t}$ were $3.8 \%$ and $2.6 \%$, respectively, and this is comparable to $1.2 \%, 3.8 \%$ and $0.6 \%$, for $g_{i l}, g_{i t}$ and $g_{i n}$ found here for the MacLachlan et al. [22] dataset and $0.2 \%, 1.4 \%$ and $3.8 \%$ for the Hooks et al. [9] dataset (Table 2). In general, for the intracellular values, the errors in the retrievals for the dataset of Hooks et al. [9] for the higher noise cases are a little larger than those for the dataset of MacLachlan et al. [22]. Finally, it would appear that the accuracy of the retrieval of the fibre rotation angle $\alpha$ lies between the accuracy of retrievals of the two types of conductivities, which is consistent with previous work [14].

\section{Discussion}

Although the solver in the first pass was always able to converge to an optimum value that gave three quite accurate extracellular conductivity values, it was usually the case that the intracellular values in the first pass were not very accurate. This was to be expected since the inversion technique is designed to take advantage of the fact that almost all of the current is in the extracellular space when 'closely-spaced' electrodes are used [23]. However, in this work, it was occasionally observed that sometimes one or two of the intracellular conductivities converged to non-physiological values in the first pass. Rather than exclude inversion runs on the basis of some arbitrary value for each conductivity, it was decided to exclude runs that had three or more parameters that were outside of the range mean \pm 1 
standard deviation (Section 3.6). This method meant that not only were the runs that resulted in non-physiological intracellular conductivities excluded, but any other runs which converged away from the 'correct' (generating) values were also excluded.

In the case of the MacLachlan et al. [22] dataset, this method resulted in the exclusion of 5, 2 and 2 runs for $1 \%, 2 \%$ and $5 \%$ noise, respectively, whereas for the Hooks et al. dataset [9] only two runs were excluded (one each for $2 \%$ and $5 \%$ noise). No exclusions were necessary for the second pass, primarily because there was a larger range of values in these cases (Table 2). It is not clear why more exclusions were necessary for the MacLachlan et al. [22] dataset, particularly as one would expect that $1 \%$ noise would provide fewer difficulties for the inversion than $5 \%$ noise, say. However, for the $1 \%$ case for this dataset there seemed to be two different optimum values to which the solver tended to converge. For reference, if all 15 inversion runs were included in the 1\% MacLachlan et al. [22] case, then the relative errors for $g_{e l}, g_{e t}$ and $g_{e n}$ would increase to $1.1 \%, 3.4 \%$ and $1.7 \%$ from $0.3 \%, 0.2 \%$ and $0.3 \%$, respectively.

This work has found that it is very important to determine the extracellular values as accurately as possible in the first pass, since they are held constant in the second pass and seem to affect the accuracy of retrievals of the intracellular conductivities in the second pass. This was why the above method was developed for excluding inaccurate runs from those used to calculate the mean values from the first pass. The method seems appropriate for experimental purposes, where it is then not necessary to make assumptions about the conductivity data that is to be retrieved. In addition, it seems practical since experiments of this sort would be designed to make multiple measurements of potential (ie. in many cardiac cycles) and some method of quality control is necessary.

In this study, certain assumptions associated with the model and the placement of the array are made. Firstly, it is assumed in the model that the imbrication angle (the angle of inclination of the fibres relative to the epicardial surface) is zero. This is a common assumption [5], which is not unreasonable in this case, since measurements will be made on the ventricular wall, where the angle is less than $5^{\circ}$. It is also common to assume that fibre rotation varies linearly with depth [5], which is applicable in this region (but not near the base or apex of the heart $[20])$. Another assumption made is that when making measurements the array is aligned with the longitudinal and transverse directions of the fibres, something that is possible using various techniques [29,32,33]. It is also assumed that no significant injury currents are caused when the array is inserted into the tissue [21]. In addition, it is also implicitly assumed that it is possible to fabricate an array with these micrometre-sized electrode spacings, which has been demonstrated by various groups (see discussion in [14] and more recently, as discussed in Section 1 $[10,1,25,24])$.

\section{Conclusions}

This work has presented an extension of a previous solution technique [15] to solve the bidomain model in a block of cardiac tissue, so that six, rather than four, cardiac conductivities can be used. An extension of a two-pass inversion technique that allows the conductivities (and fibre rotation angle) to be retrieved 
from measurements of electric potential on the heart was also presented. A new multi-electrode array is proposed, and simulated measurements of potential on the array are used, in conjunction with the inversion technique, to demonstrate that it is possible to accurately retrieve all six bidomain cardiac conductivities $\left(\mathrm{g}_{j k}, j=i, e, k=l, t, n\right)$, as well as fibre rotation angle. In fact it is found that, using the proposed array, the six conductivity values and fibre rotation could be retrieved with similar accuracy to the retrieval of the four conductivities $\left(\mathrm{g}_{j k}\right.$, $j=i, e, k=l, t)$ in the previous work [14] and, in particular, the three extracellular conductivities can be retrieved with accuracies that are about one quarter of the added noise.

This work also identifies the importance of retrieving the extracellular conductivities as accurately as possible in the first pass, since their accuracy affects the accuracy to which the intracellular conductivity values can be retrieved in the second pass. To this end, a method is suggested for eliminating inaccurate inversion runs from the first pass. An additional benefit to this method is that extremely inaccurate values for the intracellular conductivities in the first pass are also removed, resulting in more accurate initial values for the intracellular conductivities in the second pass, since they are found as the means of the first pass values.

Having shown that this array design is theoretically capable of retrieving all six bidomain cardiac conductivities, later work could consider other array designs to see if they are capable of even higher accuracy in retrieving, in particular, the intracellular conductivity values. Plans for future work also include fabricating the electrode array using micro-machining techniques and, after testing, making in vivo potential measurements in animals, during the ST segment, when the heart is isoelectric. The inversion technique, described here, would then be used to determine the six bidomain cardiac conductivity values. Such measurements could be made in various types of tissue, including normal and diseased tissue, and in various positions on the heart.

Acknowledgements This work was supported in part by a Griffith University New Researcher Grant.

\section{References}

1. Caldwell, B.J., Trew, M.L., Sands, G.B., Hooks, D.A., LeGrice, I.J., Smaill, B.H.: Three distinct directions of intramural activation reveal nonuniform side-to-side electrical coupling of ventricular myocytes. Circ Arrhythmia Electropysiol 2, 433-440 (2009)

2. Clayton, R.H., Bernus, O., Cherry, E.M., Dierckx, H., Fenton, F.H., Mirabella, L., Panfilov, A.V., Sachse, F.B., Seemann, G., Zhang, H.: Models of cardiac tissue electrophysiology: Progress, challenges and open questions. Prog. Biophys. Mol. Bio. 104(1-3), 22-48 (2011)

3. Clerc, L.: Directional differences of impulse spread in trabecular muscle from mammalian heart. J. of Physiol. 255, 335-346 (1976)

4. Foster, K.R., Schwan, H.P.: Dielectic properties of tissue and biological materials: A critical review. Crit. Rev. Biomed. Eng. 17(1), 25-104 (1989)

5. Franzone, P.C., Guerri, L., Taccardi, B.: Spread of excitation in a myocardial volume: Simulation studies in a model of anisotropic ventricular muscle activated by point stimulation. J. Cardiovasc. Electrophysiol. 4(2), 144-160 (1993)

6. Geneser, S.E., Kirby, R.M., MacLeod, R.S.: Application of stochastic finite element methods to study the sensitivity of ECG forward modeling to organ conductivity. IEEE Trans. Biomed. Eng. 55(1), 31-40 (2008)

7. Gilboa, E., Rosa, P.S.L., Nehorai, A.: Estimating electrical conductivity tensors of biological tissues using microelectrode arrays. Ann. Biomed. Eng. 40(10), 2140-2155 (2012) 
8. Graham, L.S., Kilpatrick, D.: Estimation of the Bidomain conductivity parameters of cardiac tissue from extracellular potential distributions initiated by point stimulation. Ann. Biomed. Eng. 38(12), 3630-3648 (2010)

9. Hooks, D.: Myocardial segment-specific model generation for simulating the electrical action of the heart. BioMed. Eng. OnLine 6(1), 21-21 (2007)

10. Hooks, D., Trew, M.: Construction and validation of a plunge electrode array for threedimensional determination of conductivity in the heart. IEEE Trans. Biomed. Eng. 55(2), 626-635 (2008)

11. Hooks, D.A., Tomlinson, K.A., Marsden, S.G., LeGrice, I.J., Smaill, B.H., Pullan, A.J., Hunter, P.J.: Cardiac microstructure: Implications for electrical propagation and defibrillation in the heart. Circ. Res. 91(4), 331-338 (2002). URL http://circres.ahajournals.org/cgi/content/abstract/91/4/331

12. Hooks, D.A., Trew, M.L., Caldwell, B.J., Sands, G.B., LeGrice, I.J., Smaill, B.H.: Laminar arrangement of ventricular myocytes influences electrical behavior of the heart. Circ. Res. 101(10), e103-112-e103-112 (2007)

13. Johnston, B.M., Johnston, P.R.: Possible four-electrode configurations for measuring cardiac tissue fibre rotation. IEEE Trans. Biomed. Eng. 54(3), 547-550 (2007)

14. Johnston, B.M., Johnston, P.R., Kilpatrick, D.: Analysis of electrode configurations for measuring cardiac tissue conductivities and fibre rotation. Ann. Biomed. Eng. 34(6), 986-996 (2006)

15. Johnston, B.M., Johnston, P.R., Kilpatrick, D.: A new approach to the determinination of cardiac potential distributions: Application to the analysis of electrode configurations. Math. Biosci. 202(2), 288-309 (2006)

16. Johnston, P.R.: Cardiac conductivity values - a challenge for experimentalists? NFSI \& ICBEM pp. 39-43 (2011). DOI 10.1109/NFSI.2011.5936816

17. Johnston, P.R., Kilpatrick, D.: The effect of conductivity values on ST segment shift in subendocardial ischaemia. IEEE Trans. Biomed. Eng. 50(2), 150-158 (2003)

18. Johnston, P.R., Kilpatrick, D., Li, C.Y.: The importance of anisotropy in modelling ST segment shift in subendocardial ischaemia. IEEE Trans. Biomed. Eng. 48(12), 1366-1376 (2001)

19. Kuntsevich, A., Kappel, F.: SolvOpt: The solver for Local Nonlinear Optimisation Problems, version 1.1 in C. Institute for Mathematics: Karl-Franzens University of Graz (1997)

20. LeGrice, I.J., Hunter, P.J., Smail, B.H.: Laminar structure of the heart: a mathematical model. Am. J. Physiol. 272, H2466-H2476 (1997)

21. Li, Q.: Transmyocardial ST potential distributions in ischaemic heart disease. Ph.D. thesis, University of Tasmania (2005)

22. MacLachlan, M.C., Sundnes, J., Lines, G.T.: Simulation of st segment changes during subendocardial ischemia using a realistic 3 -d cardiac geometry. IEEE Trans. Biomed. Eng. 52(5), 799-807 (2005)

23. Plonsey, R., Barr, R.C.: The four-electrode resistivity technique as applied to cardiac muscle. IEEE Trans. Biomed. Eng. 29(7), 541-546 (1982)

24. Pollard, A.E., Barr, R.C.: A biophysical model for cardiac microimpedance measurements. Am J Physiol Circ Physiol 298, H1699-H1709 (2010)

25. Pollard, A.E., Ellis, C.D., Smith, W.M.: Linear electrode arrays for stimulation and recording within cardiac tissue space constants. IEEE Trans. Biomed. Eng. 55(4), 1408-1414 (2008)

26. Potse, M., Coronel, R., Falcao, S., LeBlanc, A.R., Vinet, A.: The effect of lesion size and tissue remodeling on ST deviation in partial-thickness ischemia;. Heart Rhythm 4(2), 200-206 (2007)

27. Press, W.H., Flannery, B.P., Teukolsky, S.A., Vetterling, W.T.: Numerical Recipes, The Art of Scientific Computing, 2nd edn. Cambridge University Press, Cambridge (1992)

28. Roberts, D.E., Hersh, L.T., Scher, A.M.: Influence of cardiac fiber orientation on wavefront voltage, conduction velocity and tissue resistivity in the dog. Circ. Res. 44, 701-712 (1979)

29. Roberts, D.E., Scher, A.M.: Effects of tissue anisotropy on extracellular potential fields in canine myocardium in situ. Circ. Res. 50, 342-351 (1982)

30. Roth, B.J.: Electrical conductivity values used with the bidomain model of cardiac tissue. IEEE Trans. Biomed. Eng. 44(4), 326-328 (1997)

31. Schmitt, O.H.: Biological information processing using the concept of interpenetrating domains. In: K.N. Leibovic (ed.) Information Processing in the Nervous System, chap. 18, pp. 325-331. Springer-Verlag, New York (1969) 
32. Steendijk, P., Mur, G., van der Velde, E.T., Baan, J.: The four-electrode resistivity technique in anisotropic media: Theoretical analysis and application on myocardial tissue in Vivo. IEEE Trans. Biomed. Eng. 40(11), 1138-1147 (1993)

33. Wang, Y., Schimpf, P.H., Haynor, D.R., Kim, Y.: Geometric effects on resistivity measurements with four- electrode probes in isotropic and anisotropic tissues. IEEE Trans. Biomed. Eng. 45(7), 8-19 (1998) 